Letter to the editor

\title{
Relation between ideal and real strengths of metallic glasses
}

\author{
M.Q. Jiang ${ }^{\text {a }}$, F. Jiang ${ }^{\text {b }}$, V. Keryvin ${ }^{\text {c }}$, J.X. Meng ${ }^{\text {c }}$, J. Sun ${ }^{\text {b }}$, L.H. Dai ${ }^{\text {a,* }}$ \\ a State Key Laboratory of Nonlinear Mechanics, Institute of Mechanics, Chinese Academy of Sciences, Beijing 100190, China \\ b State Key Laboratory for Mechanical Behavior of Materials, Xi'an Jiaotong University, Xi'an 710049, China \\ c Department of Materials Engineering, LIMATB, EA4250, Université de Bretagne-Sud, Centre de recherche de St. Maudé, 56321 Lorient cedex, France
}

\section{A R T I C L E I N F O}

\section{Article history:}

Received 22 July 2012

Received in revised form 5 September 2012

Available online 5 October 2012

\section{Keywords:}

Metallic glasses;

Ideal strength;

Real strength;

Potential energy landscape;

Shear transformation zone

\begin{abstract}
A B S T R A C T
Available experimental data of about 110 metallic glasses show that the ratios of room-temperature strengths to low-temperature ideal strengths have universal upper and lower bounds. The two bounds are rationalized by taking cooperative shearing of shear transformation zone (STZ) operations into consideration in the potential energy landscape thermodynamics. It is striking to find that the real-to-ideal strength gap results from both configurational and thermo-vibrational contributions to STZs. The former determines the upper bound, while the latter further decreases strengths to the lower bound. The results may shed new insight into metallic glass strength and flow.
\end{abstract}

(c) 2012 Elsevier B.V. All rights reserved.

\section{Introduction}

The scientific interest in strength of materials could be traced back to the famous notebooks of Leonardo da Vinci [1]. However, not until the early years of the twentieth century did people relate strength of materials with their atomic structure. In 1926, Jacov Frenkel [2] calculated the ideal shear strength of a perfect fcc metal to be about a tenth of its shear modulus G. However, real crystalline materials yield strengths two to three orders of magnitude lower. This discrepancy was attributed to dislocations. The real strengths for crystalline materials have been widely estimated according to the resistance of dislocation motion such as Peierls-Nabarro force, grain size, dislocation junction, etc. [3-6]. In contrast, metallic glasses or glassy alloys, representing a young class of advanced materials, are free of dislocations [7-10]. It is highly expected that strengths of such amorphous materials could approach the theoretical limit. These materials receive therefore much attention from both scientific and engineering points of view [11-20].

In this aspect, a key finding is the intrinsic correlation of strength for inhomogeneous deformation with glass transition temperature [14, $16,17]$, which indicates the similarity between stress-driven yielding and temperature-caused glass transition in metallic glasses [16,21,22]. Actually, both physical processes are underpinned by collective motions of atomic clusters, termed shear transformation zones (STZs) [23-25]. By developing the cooperative shearing model (CSM) of STZs, Johnson and Samwer [13] proposed a universal power-law of 2/3 of temperature dependent yield strength. In particular, Cheng and Ma [18] applied this

\footnotetext{
* Corresponding author. Tel.: +86 10 82543958; fax: +8610 82543977.

E-mail addresses: mqjiang@imech.ac.cn (M.Q. Jiang), lhdai@lnm.imech.ac.cn (L.H. Dai).
}

power law to homogeneous deformation by atomistic calculations, and successfully predicted the ideal strengths for metallic glasses. Their prediction has recently been confirmed by employing an in situ TEM tension technique [20]. These intriguing progresses greatly motivate us to understand metallic glass strengths from real value to ideal/theoretical limit. In fact, their relationship has not been established theoretically up to now, which is a major scientific challenge. In this letter, we analyze the experimental data of both real and ideal strengths for more than 100 metallic glasses from 24 different alloy systems. The relationship between room-temperature (RT) and low-temperature ideal strengths is quantitatively bridged combining the potential energy landscape (PEL) theory, the CSM of STZs with fracture mechanics.

\section{Experimental observations}

Fig. 1 presents the macroscopic shear strength $\tau_{\mathrm{yT}}$ at RT versus the athermal theoretical shear strength $\tau_{0}$ for $\sim 110$ metallic glasses. Here, we roughly adopt $\tau_{\mathrm{yT}}$ as half of the yield strength $\sigma_{\mathrm{yT}}$ in monotonic loading (Tresca's yield criterion), ignoring the small normal stress dependence $[13,17,26]$. It must be pointed out that the "thermal" actually means a temperature as low as possible rather than the absolute zero temperature. Following the previous works $[16,18,27], \tau_{0}$ maybe recall the origin of $G / 10$, skirting its very small temperature dependence $[13,28,29]$. It is believed that these approximations cannot significantly change the essential physics. The experimental data for $\sigma_{\mathrm{yT}}$ and $G$ at RT can be found in the literature [13,21,30-33]. It can be observed from Fig. 1 that the real strengths are indeed quite close to their theoretical strengths, being of the same order of magnitude. The former is only a fraction smaller than the latter. Very interestingly however, all experimental data can be bounded by two straight lines. More specifically, 


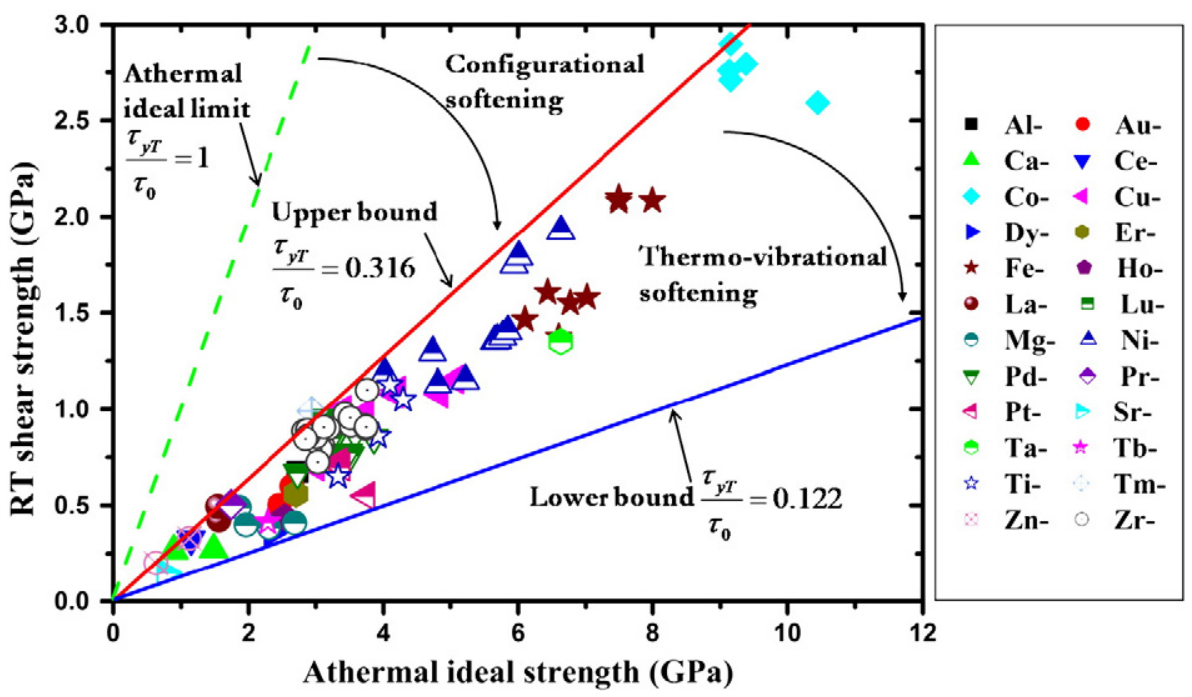

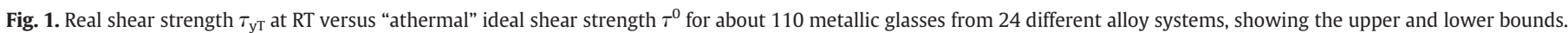


limit.

the ratio of the RT strength to the athermal theoretical strength obeys the following universal relationship:

$\Gamma_{\text {upper }} \geq \frac{\tau_{\text {yT }}}{\tau_{0}} \geq \Gamma_{\text {lower }}$

The fitting to the experimental data in Fig. 1 shows that the two bounds, $\Gamma_{\text {upper }}$ and $\Gamma_{\text {lower }}$, are very close to values of 0.316 and 0.122 , respectively. It is clear that there exists a gap between the real strengths and their ideal limit, i.e. $\tau_{\mathrm{yT}} / \tau_{0}=1$, marked by the green dashed line in Fig. 1. Some questions should arise from our observations. What is the physical meaning of the upper and lower bounds of the real strengths? Due to absence of dislocations in metallic glasses, what should be responsible for the gap of the real-ideal strengths? Undoubtedly, definitive answers originate from the unique response of atomic structures of metallic glasses to applied stress. Considering the complexity of disordered structures, we resort to the PEL theory [34-37] to reveal the underlying physics of our observed phenomena.

\section{Theory and discussion}

PEL is a multidimensional surface describing the potential energy function of a glassy system that depends on the spatial location for its constituent particles. Intuitively, the state of such a system can be well represented by a point on or above the hypersurface. By analogy to Earth's topographic maps, Stillinger and Weber [34,35] provided a formally exact portioning of the configurational space as a sum of distinct basins, associating with each local minimum of the potential energy surface, namely, an inherent structures (IS). The PEL picture provides a natural separation of the system's state into sampling distinct ISs and vibration within an IS. The PEL approach thus permits identification of the IS in an ideal metallic glass at very low temperature and a real sample at RT. As illustrated on the left in Fig. 2, the ideal sample should correspond to the IS with the lowest potential energy (deepest "megabasin") that is devoid of substantial regions with local crystalline order [38]. At a low enough temperature the ideal system becomes stuck in the single IS with almost frozen vibration, unable to surmount the highest energy barrier. As temperature increases to RT, the depth of ISs in the real sample decreases and the vibration intensifies, as shown on the right in Fig. 2. Meanwhile, the real system displays a proliferation of well-separated megabasins, corresponding to the increase of the configurational entropy. Consequently, the difference of the IS between the ideal system and the real one has the configurational and vibrational aspects. It is such difference that results in the gap of their strengths, because the strength of systems per se reflects the mechanical instability of their ISs that is normally obscured by the thermal vibration [13,39-41]. Hence, the ratio of the real-ideal strengths can be expressed as a sum of the configurational contribution $r_{\text {conf }}$ and the thermo-vibrational one $r_{\text {ther, that is }}$

$\frac{\tau_{\mathrm{yT}}}{\tau_{0}}=r_{\text {conf }}+r_{\text {ther }}$,

where $r_{\text {conf }}=\left[\tau_{\mathrm{y} 0} / \tau_{0}\right]_{\text {conf }}$ measures the ratio between the ideal strengths and the real strengths at the athermal limit, and $r_{\text {ther }}=$ $\left[\tau_{\mathrm{yT}} / \tau_{\mathrm{y} 0}\right]_{\text {ther }}$ denotes the thermal softening effect on the real strengths due to temperature increase from very low temperature to RT. Next, the main task is to quantitatively determine the configuration and vibration terms in Eq. (2).

Recent studies have identified solute-centered polyhedra as the fundamental building blocks of metallic glasses, constituting shortrange-order; these polyhedra subsequently pack together to fill threedimensional space obeying a certain rule, giving rise to medium-rangeorder [42-45]. Driven by external stress, the short-to-medium-range order could be lost via STZ operations. Therefore, it is reasonable to treat the virgin metallic glass as a composite consisting of potential STZ sites within the elastic confinement of a surrounding matrix [23,46]. Here we consider a semi-infinite metallic glass plate containing an edge-crack subject to a remote shear stress. The edge-crack has finite size that is so small

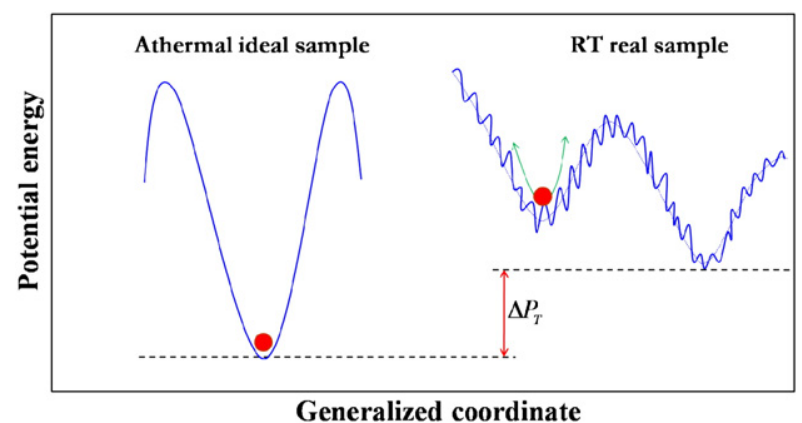

Fig. 2. Schematic illustration of archetypal potential energy landscapes of athermal ideal and RT real metallic glass. 
and can not affect the macroscopic strength. The concentrated stress at the crack tip would preferentially activate the STZ transitions along the crack path, but which relies heavily on the spatial configuration of these potential STZ sites and their activity. Subsequently, it is very interesting to see how these STZ sites respond to the external stress for the ideal and real samples schematized in Fig. 2 and what finally dominates their strengths.

In terms of the PEL, the individual STZ operation confined within the elastic medium can be viewed as a sub-IS hopping event that corresponds to the fast $\beta$ relaxation process $[13,39,47]$. It has been recognized that both $\beta$ relaxations and STZs are related to atomic structural heterogeneity in metallic glasses; their activation energies or probabilities are nearly the same [48-50]. The STZ operations would occur preferentially at the heterogeneous sites where the $\beta$ relaxations are also strong. The cascade and avalanche of a series of STZs would induce an intra-IS hopping event, associated with the slower $\alpha$ relaxation process $[13,39,40]$. The plastic irreversible $\alpha$ hopping event at large scale will directly contribute to macroscopically perceptive plasticity. The PEL of the ideal glass shown on the left in Fig. 2 implies a quite homogeneous atomic structure, in which structural heterogeneities are very low in population and very small in size. It is then expected that the $\beta$ relaxations within the ideal IS are extremely obscure, especially at low enough temperature. As a result, under the remote stress, the potential STZ sites are unlikely to mature to true STZs. In other words, these STZs only behave with individuality and nonlinear elasticity due to the confinement of surrounding elastic matrix. See the inset (a) of Fig. 3. In order to simultaneously activate elastic operation of such STZs and drive the crack propagation, the stress field at the crack tip must maintain a very high level, near to theoretical strength. In this way, the ideal system can escape from the deepest megabasin (the left in Fig. 2), corresponding to material failure. Before that, the entire sample deforms elastically and homogeneously without the intervention of shear bands. However, for the RT real sample shown on the right in Fig. 2, the case is just opposite. Due to obvious structural defects and relatively pronounced $\beta$ relaxations, the potential STZ sites can grow and mature, preferentially along the crack path. These matured STZs have a much larger probability to interact with each other, and avalanche due to topologically instability. The percolation of these unstable STZs finally leads to the local collapse of the confining matrix and breakdown of elasticity [the inset (b) to Fig. 3]. When the local percolation of STZs reaches a critical limit, macroscopic plastic yielding occurs via shear-banding $[13,46]$. This process can be understood in the PEL picture as the

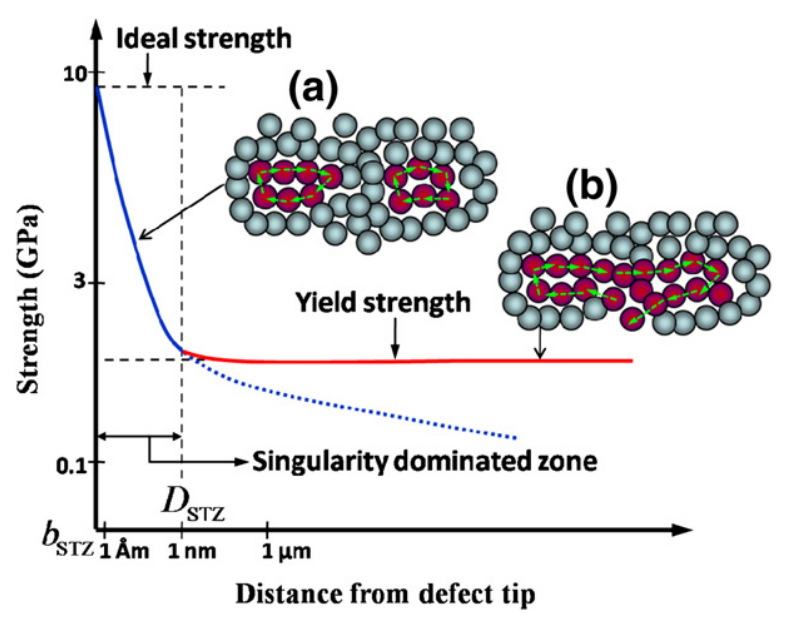

Fig. 3. Strength as a function of the distance from a representative atom at a crack-like defect tip. Inset: (a) the STZs exhibit individuality and nonlinear elasticity on the scale $b_{\mathrm{STZ}} \sim 10^{-10} \mathrm{~m}$ of the lattice distortion of potential STZ site; (b) one STZ can interact with another over the scale $D_{\mathrm{STZ}} \sim 10^{-9} \mathrm{~m}$ of the spacing of potential STZ sites, where the local flow event occurs. transition of the system state from one IS to another with more depth, but crossing a relatively low potential energy barrier.

According to the PEL description of STZs above, it can be suggested that the fundamental process for plastic flow in real metallic glasses is discrete, consisting of at least two important length scales. One is the magnitude of the lattice-like distortion of potential STZ site that should be at the atomic scale, denoted by $b_{\mathrm{STZ}} \sim 10^{-10} \mathrm{~m}$. This quantity is similar to the Burgers vector in crystals, but without the directionality here. The other is the spacing of potential STZ sites, $D_{\mathrm{STZ}} \sim 10^{-9} \mathrm{~m}$, considering the size of STZs [16,51-53] and dense packing configuration for metallic glass structures $[42,43,54]$. On the $b_{\mathrm{STZ}}$ scale, the STZs exhibit individuality, as shown in the insert (a) to Fig. 3. The probability to blunt a crack front should be extremely small. Consequently, a crack that does not undergo plasticity will remain sharp, advancing by atomic decohesion. Until one STZ interacts with another over the $D_{\text {STZ }}$ scale, the plastic flow event occurs, as schematically depicted in the insert (b) to Fig. 3. The STZ behaviors on the two length scales just correspond to what happens in the ideal and real samples, respectively. Such a process can be approximately understood in terms of strength varying with the distance $r$ from a representative atom at the crack tip, sketched in Fig. 3. It is well known that, before the plastic yielding, the $1 / \sqrt{r}$ singularity dominates the stress filed $\tau_{r}^{K}$ measured by the Mode II stress intensity factor $K_{\mathrm{II}}$ [55]. Hence, it is reasonable to assume that the theoretical shear strength, i.e. the largest possible stress to overcome the bond strength, would be approached at $r=b_{\mathrm{STZ}}$, and the macroscopic ideal strength $\tau_{0}$ should be in proportion to the theoretical shear strength at the cluster scale, which has been confirmed previously [56]. Therefore, we can obtain a relationship:

$\tau^{0} \propto \tau_{r=b_{\mathrm{STZ}}}^{K} \propto \frac{K_{\mathrm{II}}}{\sqrt{2 \pi b_{\mathrm{STZ}}}}$.

Over a distance of $D_{\mathrm{STZ}} \sim 10^{-9} \mathrm{~m}$, the stress decays to the yield strength that can be still determined by the K-field,

$\tau_{\mathrm{y} 0} \propto \tau_{r=D_{\mathrm{STZ}}}^{K} \propto \frac{K_{\mathrm{II}}}{\sqrt{2 \pi D_{\mathrm{STZ}}}}$.

It must be pointed out that the singularity dominated zone provides an elastic medium through which the stress declines rapidly, matching the high theoretical strength $\tau^{0}$ on one side, and the low yield stress $\tau_{\mathrm{y} 0}$ on the other. Note that the fracture mechanics analysis only considers the configurational aspect. Combining Eq. (3) with Eq. (4) obeys the following intrinsic correlation:

$r_{\text {conf }}=\left[\frac{\tau_{\mathrm{y} 0}}{\tau_{0}}\right]_{\text {conf }} \propto \sqrt{\frac{b_{\mathrm{STZ}}}{D_{\mathrm{STZ}}}} \sim \frac{1}{\sqrt{10}} \approx \Gamma_{\text {upper }}$.

This correlation is totally identical to the upper bound line fitted by experimental data, i.e., the red solid line in Fig. 1. It implies that $10 b_{\text {STZ }}$ could the upper limit of the STZ spacing. That actual spacing should be lower than it, leading to further decrease of real strengths. It is also noted that the value $r_{\text {conf }}$ is very close to the ratio between the athermal strength predicted from inhomogeneous deformation and that derived from homogeneous deformation in metallic glasses [18]. Now it can be concluded that the gap between the athermal ideal strengths and the upper bound of the real strengths is bridged quantitatively, which is attributed to the deformation mode change from elastically homogeneous to plastically inhomogeneous (shearbanding). This strength decrease due to the deformation mode change has only a configurational origin.

As for the thermo-vibrational softening effect on the real strengths, Johnson and Samwer [13] have already given a description based on 
the CSM of STZs. The thermo-vibrational softening term in Eq. (2) can be precisely expressed as [13]

$r_{\text {ther }}=-\left[\frac{k_{\mathrm{B}}}{W_{T_{g}}} \cdot \frac{G_{0 T}}{G_{0 T_{g}}} \ln \left(\omega_{0} / C \dot{\gamma}\right) T\right]^{2 / 3}$,

where $k_{\mathrm{B}}$ is the Boltzmann constant, $W_{T_{\mathrm{g}}}$ is the local megabasin barrier of real samples at the glass transition temperature $T_{\mathrm{g}}, G_{0 T} / G_{0 T_{\mathrm{g}}}$ is the ratio of zero-stress shear moduli at $T(\mathrm{RT}, \sim 300 \mathrm{~K})$ and $T_{\mathrm{g}}$, respectively, $\omega_{0}$ is the attempt frequency, $C$ is a dimensionless constant of order unity, and $\dot{\gamma}$ is the applied shear strain rate. Eq. (6) essentially describes the dependence of the temperature on the IS barrier energy. Considering the experimental data in Fig. 1 that are extracted from the quasi-static cases, $\ln \left(\omega_{0} / C \dot{\gamma}\right)^{\sim} 30$ [13]. For inhomogeneous deformation, $W_{T_{\mathrm{g}}}$ is chosen to be about $1.4 \times 10^{-18} \mathrm{~J}[13,20]$. Again, ignoring the weak temperature dependence, $G_{0 T} / G_{0 T_{\mathrm{g}}} \sim 1$. Therefore, $r_{\text {ther }}$ is calculated to be about -0.194 . Inserting this value into Eq. (2) and joining with Eq. (5), we obtain the expression of the lower bound line (i.e., the blue solid line in Fig. 1) for the RT real strengths:

$\frac{\tau_{\mathrm{yT}}}{\tau_{0}}=r_{\text {conf }}+r_{\text {ther }}=\frac{1}{\sqrt{10}}-0.194=\Gamma_{\text {lower }}$.

The remarkable agreement between the theoretical predictions [Eqs. (5) and (7)] and the empirical fitting lines confirms that the ideal-real strengths gap results from the configurational and thermovibrational contributions to cooperative shearing of STZs. In fact, the two contributions are highly coupled and strongly material dependent, because the experimental data scatter in a narrow region, instead of following one single line. If the configurational softening dominates, the real strengths can approach the upper limit that is predicted by Eq. (5). Additional thermo-vibrational softening makes the strengths decrease furthermore. Their lower bound is given by Eq. (7), which corresponds to the strongest thermo-vibrational softening.

Finally, we briefly discuss related works. Very recently, the ideal strength of a $\mathrm{Cu}-\mathrm{Zr}$ metallic glass at RT has been achieved by Tian et al. [20] through consciously choosing sample size and loading technique. In their experiments, materials deform mainly elastically (initial linear and followed nonlinear) and plastic deformation is very small, during which shear-banding does not occur. In fact, this sample state should be very close to that of an ideal glass shown on the left in Fig. 2, just at RT rather than low temperature. The resultant deformation mechanism can be illustrated by the insert (a) to Fig. 3. Similar deformation mode transition and ideal strengths have also been obtained in probing very small volumes of metallic glasses that are likely to be defect free [12,57-59]. Furthermore, we can envision an attractive situation, in which the potential STZ sites can homogeneously and abundantly distribute in samples, having the chance to grow into true STZs. It is then expected that not only higher strength but also larger plasticity would be obtained before sudden failure of materials [59-61]. Yu et al. [50] have recently reported a La-based metallic glass which shows significant tensile plasticity. Their observations reveal that plenty of nanoscale heterogeneous structures are distributed homogeneously in this material with strong $\beta$ relaxations. Such a structure facilitates simultaneous maturation of potential STZs that self-organize into multiple shear bands to accommodate large plastic deformation. In addition, it is well known that decreasing temperature can make metallic glass strengths increase [62-64]. This strengthening effect should contribute both to the decrease of IS depth and the weakness of $\beta$ relaxations.

\section{Conclusions}

In conclusion, the upper and lower bounds of RT real strengths and their relationship with the "athermal" ideal limit for metallic gasses were investigated, which could be characterized in terms of PEL of cooperative shearing of STZs. The upper bound is determined by the configurational softening due to the deformation mode transition from homogeneous to inhomogeneous. The thermo-vibrational softening further decreases strengths from the upper to lower bound. We believe that the strength softening mechanism revealed in the present work could be generalized to nonmetallic glasses (oxides, polymers, etc.) and even crystalline solids although both $r_{\text {conf }}$ and $r_{\text {ther }}$ may vary.

Supplementary data to this article can be found online at http:// dx.doi.org/10.1016/j.jnoncrysol.2012.09.016.

\section{Acknowledgments}

M.Q.J. is very grateful to Professor E. Ma and Professor W. H. Wang for valuable discussions. Financial support is from the NSFC (Grant Nos. 11002144, 11023001, 11132011 and 11021262), the National Natural Science Foundation of China-NSAF Grant No: 10976100 and the National Key Basic Research Program of China (Grant Nos. 2009CB724401 and 2012CB937500). This work is also partially supported by the State Key Laboratory for Mechanical Behavior of Materials, Xi'an Jiaotong University. We are highly grateful to the anonymous reviewer for his/her helpful comments, which have improved our article significantly.

\section{References}

[1] S.P. Timoshenko, History of Stength of Materials, McGraw-Hill Book Company, Inc., New York, 1953.

[2] J. Frenkel, Z. Phys. 37 (1926) 572.

[3] J.P. Hirth, J. Lothe, Theory of Dislocations, Kreiger, Malabar, FL, 1992.

[4] D. Roundy, C.R. Krenn, M.L. Cohen, J.W. Morris Jr., Phys. Rev. Lett. 82 (1999) 2713.

[5] V.B. Shenoy, R.V. Kukta, R. Phillips, Phys. Rev. Lett. 84 (2000) 1491.

[6] D.M. Clatterbuck, C.R. Krenn, M.L. Cohen, J.W. Morris, Phys. Rev. Lett. 91 (2003) 135501.

[7] W.I. Klement, R.H. Willens, P. Duwez, Nature 187 (1960) 869.

[8] A.L. Greer, Science 267 (1995) 1947.

[9] C.J. Byrne, M. Eldrup, Science 321 (2008) 502.

[10] A.R. Yavari, A. Le Moulec, A. Inoue, N. Nishiyama, N. Lupu, E. Matsubara, W.J. Botta, G. Vaughan, M. Di Michiel, A. Kvick, Acta Mater. 53 (2005) 1611.

[11] W.L. Johnson, MRS Bull. 24 (1999) 42.

[12] H. Bei, Z.P. Lu, E.P. George, Phys. Rev. Lett. 93 (2004) 125504

[13] W.L. Johnson, K. Samwer, Phys. Rev. Lett. 95 (2005) 195501.

[14] B. Yang, C.T. Liu, T.G. Nieh, Appl. Phys. Lett. 88 (2006) 221911.

[15] A.L. Greer, E. Ma, MRS Bull. 32 (2007) 611.

[16] M.W. Chen, Annu. Rev. Mater. Res. 38 (2008) 445.

[17] Y.H. Liu, C.T. Liu, W.H. Wang, A. Inoue, T. Sakurai, M.W. Chen, Phys. Rev. Lett. 103 (2009) 065504.

[18] Y.Q. Cheng, E. Ma, Acta Mater. 59 (2011) 1800.

[19] L. Wondraczek, J.C. Mauro, J. Eckert, U. Kuhn, J. Horbach, J. Deubener, T. Rouxel, Adv. Mater. 23 (2011) 4578.

[20] L. Tian, Y.Q. Cheng, Z.W. Shan, J. Li, C.C. Wang, X.D. Han, J. Sun, E. Ma, Nat. Commun. 3 (2012) 609.

[21] W.H. Wang, J. Appl. Phys. 110 (2011) 053521

[22] K. Georgarakis, M. Aljerf, Y. Li, A. LeMoulec, F. Charlot, A.R. Yavari, K. Chornokhvostenko, E. Tabachnikova, G.A. Evangelakis, D.B. Miracle, A.L. Greer, T. Zhang, Appl. Phys. Lett. 93 (2008) 031907.

[23] A.S. Argon, Acta Metall. 27 (1979) 47.

[24] M.L. Falk, J.S. Langer, Phys. Rev. E 57 (1998) 7192.

[25] M.L. Falk, Science 318 (2007) 1880.

[26] J. Caris, J.J. Lewandowski, Acta Mater. 58 (2009) 1026.

[27] F. Spaepen, Nat. Mater. 5 (2006) 7.

[28] Z.Y. Zhang, V. Keppens, T. Egami, J. Appl. Phys. 102 (2007) 123508.

[29] Z.B. Lu, J.G. Li, H. Shao, H. Gleiter, X. Ni, Appl. Phys. Lett. 94 (2009) 091907.

[30] J.F. Wang, R. Li, N.B. Hua, T. Zhang, J. Mater. Res. 26 (2011) 2072.

[31] J.F. Wang, R. Li, N.B. Hua, L. Huang, T. Zhang, Scr. Mater. 65 (2011) 536.

[32] C.C. Yuan, X.K. Xi, J. Appl. Phys. 109 (2011) 033515.

[33] W.H. Wang, Prog. Mater. Sci. 57 (2012) 487.

[34] F.H. Stillinger, T.A. Weber, Phys. Rev. A 25 (1982) 978.

[35] F.H. Stillinger, T.A. Weber, Science 225 (1984) 983.

[36] C.A. Angell, Nature 393 (1998) 521.

[37] P.G. Debenedetti, P.H. Stillinger, Nature 410 (2001) 259.

[38] F.H. Stillinger, Science 267 (1995) 1935.

[39] J.S. Harmon, M.D. Demetriou, W.L. Johnson, K. Samwer, Phys. Rev. Lett. 99 (2007) 135502.

[40] G. Gagnon, J. Patton, D.J. Lacks, Phys. Rev. E 64 (2001) 051508.

[41] J.C. Dyre, N.B. Olsen, Phys. Rev. E 69 (2004) 042501.

[42] D.B. Miracle, Nat. Mater. 3 (2004) 697.

[43] H.W. Sheng, W.K. Luo, F.M. Alamgir, J.M. Bai, E. Ma, Nature 439 (2006) 419.

[44] D. Ma, A.D. Stoica, X.-L. Wang, Nat. Mater. 8 (2009) 30.

[45] A. Hirata, P.F. Guan, T. Fujita, Y. Hirotsu, A. Inoue, A.R. Yavari, T. Sakurai, M.W. Chen, Nat. Mater. 10 (2011) 28 
[46] L. Sun, M.Q. Jiang, L.H. Dai, Scr. Mater. 63 (2010) 943.

[47] G.P. Johari, M. Goldstein, J. Chem. Phys. 53 (1970) 2372.

48] S.G. Mayr, Phys. Rev. Lett. 97 (2006) 195501.

49] H.B. Yu, W.H. Wang, H.Y. Bai, Y. Wu, M.W. Chen, Phys. Rev. B 81 (2010) 220201 (R).

[50] H.B. Yu, X. Shen, Z. Wang, L. Gu, W.H. Wang, H.Y. Bai, Phys. Rev. Lett. 108 (2012) 015504.

[51] D. Pan, A. Inoue, T. Sakurai, M.W. Chen, PNAS 105 (2008) 14769

[52] C.A. Schuh, T.C. Hufnagel, U. Ramamurty, Acta Mater. 55 (2007) 4067.

[53] F. Jiang, M.Q. Jiang, H.F. Wang, Y.L. Zhao, L. He, J. Sun, Acta Mater. 59 (2011) 2057

[54] J.D. Bernal, Nature 185 (1960) 68

[55] T.L. Anderson, Fracture Mechanics: Fundamentals and Applications, Third Edition Taylor \& Francis Group, Lodon, 2005.

[56] C.A. Schuh, A.C. Lund, Nat. Mater. 2 (2003) 449
[57] W.J. Wright, in: Shear Band Processes in Bulk Metallic Glasses, Stanford University, Stanford University, 2003.

[58] A.R. Yavari, K. Georgarakis, W.J. Botta, A. Inoue, G. Vaughan, Phys. Rev. B 82 (2010) 172202

[59] D. Jang, J.R. Greer, Nat. Mater. 9 (2010) 215

[60] H. Guo, Nat. Mater. 6 (2007) 735.

[61] Z.W. Shan, J. Li, Y.Q. Cheng, A.M. Minor, S.A.S. Asif, O.L. Warren, E. Ma, Phys. Rev. B 77 (2008) 155419

[62] H.Q. Li, G. Fan, K.X. Tao, H. Choo, P.K. Liaw, Adv. Mater. 18 (2006) 752

[63] E.D. Tabachnikova, A.V. Podol'skii, V.Z. Bengus, S.N. Smirnov, D.V. Luzgin, A. Inoue, Low Temp. Phys. 34 (2008) 675

[64] V. Keryvin, K.E. Prasad, Y. Gueguen, J.C. Sangleboeuf, U. Ramamurty, Philos. Mag. 88 (2008) 1773. 